\title{
Sensitization to Epithelial Antigens in Chronic Mucosal Inflammatory Disease

\author{
Characterization of Human Intestinal Mucosa-derived Mononuclear Cells Reactive \\ with Purified Epithelial Cell-associated Components In Vitro
}

\author{
James K. Roche, Claudio Fiocchi, and Kenneth Youngman \\ Division of Gastroenterology, Department of Medicine, Duke University Medical Center, Durham, North Carolina 27710; \\ Departments of Molecular and Cellular Biology and Gastroenterology, The Cleveland Clinic Foundation, Cleveland, Ohio 44106
}

\section{Abstract}

To explore the auto-reactive potential of cells infiltrating the gut mucosa in idiopathic chronic inflammatory bowel disease, intestinal lamina propria mononuclear cells (LPMC) were isolated, characterized morphologically and phenotypically, and evaluated for antigen-specific reactivity. The last was assessed by quantitating LPMC cytotoxic capabilities against purified, aqueous-soluble, organ-specific epithelial cell-associated components (ECAC) characterized previously.

Enzyme-isolated inflammatory bowel disease LPMC were constituted primarily by $T$ lymphocytes (57 $\pm 12 \%$ OKT 3positive), B lymphocytes (18 $\pm 9 \%$ surface immunoglobulinpositive), and macrophages (11 $16 \%$ esterase-positive), and were responsive to phytohemagglutinin (mean uptake 86,933 $\mathrm{cpm} / 5 \times 10^{5}$ cells). LPMC present in abnormal segments from $71 \%$ of patients with chronic inflammatory bowel disease were cytotoxic for ECAC isolated from colon (12.5 $\pm 8.9 \%$ specific lysis) and small bowel (7.1 $\pm 6.5 \%)$, but not for kidney control antigen $(0.8 \pm 1.1 \%)$ isolated in a manner analogous to that used for ECAC $(P<0.02)$. In contrast, despite comparable responses to phytohemagglutinin (mean uptake $59,200 \mathrm{cpm} / 5$ $\times 10^{5}$ cells), LPMC from histologically normal mucosa of patients with benign (adult megacolon, Hirshsprung's disease, diverticulosis) or malignant disease failed to lyse indicator cells labeled with colon-derived ECAC $(0.3 \pm 0.08 \%)$, small bowelderived ECAC $(0.4 \pm 1.11 \%)$, or kidney antigen $(0.29 \pm 0.79 \%)$. LPMC reactivity for individual gel-purified macromolecules of small bowel-derived ECAC (designated as the "P" series of components) was greatest against component P1 (by 2-3-fold), but was detectable against three other purified components as well. The addition of patient's serum did not enhance cytotoxicity to ECAC. Characterization of the cytotoxic cell showed that it was nonadherent to plastic surfaces, bore $T$ lymphocyte-specific markers detectable by OKT 11 and OKT 3 monoclonal antibodies, and could be depleted by removal of cells with receptors for sheep erythrocytes. ECAC-specific reactivity was markedly reduced (>93\%) in most experiments when LPMC were preincubated for $1 \mathrm{~h}$ with ECAC.

These data support the concept that autosensitization to several epithelial cell-associated components has occurred in patients with chronic inflammatory bowel disease, and provide

Address reprint requests to Dr. Roche, Box 3252, Duke University.

Received for publication 27 March 1984 and in revised form 24 September 1984.

J. Clin. Invest.

(c) The American Society for Clinical Investigation, Inc.

0021-9738/85/02/0522/09 \$1.00

Volume 75, February 1985, 522-530 initial evidence that antigen-specific, cell-mediated mechanisms may play a role in the pathogenesis of these disorders.

\section{Introduction}

The process of immune sensitization at the gastrointestinal mucosal level is structurally unique $(1,2)$, but, functionally, poorly understood. In view of the large number of dietary and environmental antigens in the lumen of mucosa-bearing organs, for example, it is not clear how the sensitization process is regulated in a way conducive to the development of the most appropriate response for a particular antigenic stimulus. For instance, what signals determine whether a given response is primarily cell mediated or humoral; whether priming remains local or becomes systemic; and whether antigen-specific tolerance or immunity occur. A detailed knowledge of the factors governing the sensitization process is particularly important in the case of disorders characterized by chronic inflammation in mucosa, where immune responses harmful to mucosal epithelial cells have been described (3-5). However, most studies of the local inflammatory infiltrate in such diseases have focused on an evaluation of cell number and general immunological function (6-8).

We took a different approach to the question of aberrant sensitization at mucosal surfaces through the study of antigenspecific immune responses. We have previously shown that the immune response to gut-associated (salivary) glycoproteins is $\mathrm{T}$ lymphocyte-dependent, despite the resemblance of these macromolecules to $\mathrm{T}$-independent antigens by virtue of the repeating nature of their surface epitopes, and that the carbohydrate sidechains, rather than the core apoprotein, are the principal immunogenic moiety $(9,10)$. Analogous components have now been isolated from intestinal epithelium, purified to homogeneity, and immune (primarily humoral) reactivity to them defined at the peripheral blood level in patients with chronic inflammatory intestinal diseases $(11,12)$. An important objection to such studies, however, is the probable changed nature of the immune response and of the events in the preceding sensitization as well, when one evaluates immunological events at the mucosal rather than at the peripheral blood level: immune mechanisms detected at the periphery may be nonoperative or irrelevant at the mucosal level. In addition, circulating factors with immune regulatory potential such as immunoglobulins, lymphoid cells, and lymphokines may influence mucosal immunological reactivity. Insight into these and other mechanisms for initiation and modulation of antigen-specific reactions in mucosa can best be gained by direct evaluation of immune competent cells populating the intestine itself. Such evaluation has become possible through recently developed techniques for isolating purified mononu- 
clear cells from the intestinal lamina propria of normal and abnormal mucosa obtained by surgical resection or biopsy (6$8,13)$.

In this communication, we report that lamina propria mononuclear cells (LPMC), ${ }^{1}$ isolated from intestine involved by a chronic inflammatory disease, but not from control tissue, exhibit cytotoxicity specific for purified surface components (ECAC) of intestinal epithelium. Characterization of the effector cell suggests that it is a $T$ cell because ECAC-specific reactivity was significantly enhanced by enrichment in cells bearing surface markers of the OKT 3 and OKT 11 phenotype and was practically abolished by depletion of sheep erythrocyte (SRBC) receptor-positive cells.

\section{Methods}

Inflammatory bowel disease patients. 31 patients, 19 males and 12 females, 19-55 yr of age and undergoing intestinal resection at the Cleveland Clinic Foundation Hospital between September, 1981 and January, 1984 were studied under code. All satisfied the criteria for diagnosis developed by Truelove and Witts (14) for ulcerative colitis (12 patients), and by Schachter and Kirsner (15) for Crohn's disease (19 patients). Histological sections were available to confirm the diagnosis in each case. According to criteria established by deDombal et al. (16), disease activity was classified as mild in two, moderate in 21 , and severe in eight patients. Of the Crohn's disease patients, three had ileitis, 10 had ileocolitis, and six had colitis only. At the time of resection, 10 patients were receiving steroids (10-40 mg Prednisone/ d) plus sulfasalazine; 17 patients were receiving steroids only (5-45 mg Prednisone/d); one patient was taking Azothiaprine $(100 \mathrm{mg} / \mathrm{d})$, and three patients were receiving no specific therapy. Nevertheless, by their reactivity to the lectin phytohemagglutinin (PHA), mucosal mononuclear cells from inflammatory bowel disease patients were comparable to those from controls (see below).

Control patients. 10 patients, five males and five females, 1-74 yr of age, without chronic idiopathic inflammatory bowel disease but undergoing intestinal resection for malignant and other nonmalignant disease, were studied. Only macroscopically and histologically normal mucosa was used, with cells processed under code in an identical manner and during the same time period as specimens from inflammatory bowel disease patients. Seven patients had adenocarcinoma (CA) of the large bowel, including four with Dukes B, two Dukes C, and one Dukes $\mathrm{D}$ disease. Three patients had benign disease (adult megacolon, Hirshsprung's disease, diverticulosis). This project was approved by the Research Projects Committee of The Cleveland Clinic Foundation (1981).

Isolation of human intestinal LPMC. Representative areas of inflamed mucosa from Crohn's disease or ulcerative colitis intestine and macroscopically normal mucosa from control intestine were obtained. LPMC were isolated by the method of Bull and Bookman (6) with some modifications (8), as shown in Fig. 1. Mucosal fragments were digested over $12-16 \mathrm{~h}$ in the presence of $0.01 \%$ Clostridium histolyticum collagenase (CLS III) and $0.01 \%$ deoxyribonuclease (both from Worthington Diagnostic Systems, Inc., Freehold, NJ), and LPMC were purified over a gradient of Ficoll-Hypaque (density 1.078) spun for $5 \mathrm{~min}$ at $2,250 \mathrm{~g}$.

Depletion of adherent cells. LPMC, at 5-10 $\times 10^{6}$ cells $/ \mathrm{ml}$, were layered onto plastic tissue culture dishes (Corning Glass Works, New York, NY) and incubated for $2 \mathrm{~h}$ at $37^{\circ} \mathrm{C}$ in a $5 \% \mathrm{CO}_{2}-95 \%$ air

1. Abbreviations used in this paper: $\mathrm{CA}$, adenocarcinoma; $\mathrm{E} / \mathrm{T}$, effector to target; ECAC, epithelial cell-associated components; ECAC-C and ECAC-SB, ECAC from colon and small bowel, respectively; FCS, fetal calf serum; LPMC, lamina propria mononuclear cells; NK, natural killer; PHA, phytohemagglutinin; SRBC, sheep erythrocytes. humidified atmosphere. Unattached cells were then removed, washed, and suspended in culture medium. For purposes of this paper, these nonadherent cells will be defined as adherent cell-depleted LPMC; they contained $2 \pm 1.5 \%$ macrophages, as determined by nonspecific esterase staining (17).

Monoclonal antibody-panning technique for $T$ cell and natural killer (NK) cell enrichment of LPMC. As a modification of the technique described by Wysocki and Sato (18), $10 \mathrm{ml}$ of a goat antimouse immunoglobulin solution (Southern Biotechnology Assoc., Birmingham, AL), containing $10 \mu \mathrm{g}$ antibody $/ \mathrm{ml}$ of Tris-buffered saline at $\mathrm{pH} 9.5$, were added to a $100 \times 20$-mm bacteriological grade plastic dish (Optilux, Becton-Dickinson \& Co., Oxnard, CA). The plates were incubated at room temperature for $1 \mathrm{~h}$, washed five times, and stored at $4^{\circ} \mathrm{C}$ for at least $30 \mathrm{~min}$ before use.

Adherent cell-depleted LPMC were washed two times in phosphatebuffered saline (PBS) with 5\% fetal calf serum (FCS) and resuspended at $20 \times 10^{6}$ cells $/ \mathrm{ml}$ in the same buffer. OKT 11 or Leu $11 \mathrm{~b}$ monoclonal antibodies (Ortho Immunobiological Research Div., Raritan, NJ; Becton-Dickinson \& Co., Research Div., Mt. View, CA) were added at a 1:100 and a 1:250 dilution, respectively, to the cell suspension and incubated at $4^{\circ} \mathrm{C}$ for $30 \mathrm{~min}$, with gentle agitation every $10 \mathrm{~min}$. The monoclonal antibody-coupled LPMC were then pelleted $(200 \mathrm{~g}, 10$ $\min$ at $4^{\circ} \mathrm{C}$ ) and resuspended at $6 \times 10^{6}$ cells $/ \mathrm{ml}$ of PBS with $5 \%$ FCS. 20-30 $\times 10^{6}$ cells were dispersed slowly onto a goat anti-mouse Igcoated plate and incubated at $4^{\circ} \mathrm{C}$. After $1 \mathrm{~h}$, nonadherent cells were removed by gentle pipetting. Adherent cells were rinsed with PBS containing 5\% FCS and removed by several washes performed by gentle pipetting with the same buffer. Both adherent and nonadherent cells were assessed for purity by immunofluorescence (see below): they were found to contain $96 \pm 1.7$ and $23 \pm 8 \% \mathrm{~T}$ lymphocytes, respectively, after OKT 11 treatment. Low numbers of NK cells in unfractionated LPMC $(1.3 \pm 1.1 \%)$ were further reduced in the nonadherent fraction $(0.5 \pm 0.7 \%)$, but were unchanged in the adherent fraction $(1.0 \pm 0.86 \%)$.

$S R B C$ rosetting for $T$ cell depletion of $L P M C$. To obtain cell preparations depleted of intestinal lamina propria $\mathrm{T}$ lymphocytes, $\mathrm{E}$ rosette formation followed by Ficoll-Hypaque gradient centrifugation was carried out using SRBC pretreated with aminoethylisothiouronium bromide (Aldrich Chemical Co., Milwaukee, WI) (19). The cells at the interface were washed, suspended in medium, and assessed for cell type by immunofluorescence (see below); $11.6 \pm 5.6 \%$ were $T$ lymphocytes.

Monoclonal antibody and surface immunoglobulin methods for cell identification. Unfractionated and fractionated LPMC, at a concentration of $5 \times 10^{6}$ cells $/ \mathrm{ml}$, were assayed by resuspending the cells in PBS with $1 \%$ BSA, $2 \%$ human AB serum, and $0.2 \%$ sodium azide. Two $200-\mu \mathrm{l}$ aliquots were prepared, one receiving $5 \mu \mathrm{l}$ of monoclonal antibody (OKT 3 or Leu $11 \mathrm{~b}$ ), the other $5 \mu \mathrm{l}$ of medium as a reagent control. Cells were mixed thoroughly and placed in an ice-water bath for $30 \mathrm{~min}$ and agitated every $10 \mathrm{~min}$. At the end of the incubation time, the cells were washed twice with $2 \mathrm{ml}$ of cold PBS/5\% FCS. After the second wash, the supernatant was removed, leaving the cell pellet plus $\sim 100 \mu \mathrm{l}$ of medium. $100 \mu \mathrm{l}$ of diluted (1:20) fluorescein isothiocyanate-conjugated goat anti-mouse IgG was added to each tube, and these were incubated and washed as above. LPMC contained in the final pellet were resuspended in one drop of PBS with $30 \%$ glycerol and $0.2 \%$ sodium azide. The cells were maintained at $4^{\circ} \mathrm{C}$ until a differential count of 200 cells was performed with an Olympus model BH-RFLW microscope equipped for epiflorescence (excitation wavelength $530 \mathrm{~nm}$ ). For quantitation of surface immunoglobulinpositive cells, a similar immunofluorescence technique was utilized except that fluorescein-labeled anti-human polyvalent goat immunoglobulin (dilution, 1:10; Meloy Laboratories Inc., Springfield, VA) was used instead of monoclonal antibody in a single incubation step, with cells directly examined under fluorescence:

Mitogen-induced proliferative reactivity of LPMC. LPMC were suspended at $5 \times 10^{5}$ cells/ml of RPMI 1640 with $10 \%$ human AB serum and dispensed in triplicate into round-bottom wells in a 96-U microtiter plate (Costar, Cambridge, MA). Phytohemagglutinin (PHA- 
M, Difco Laboratories, Detroit, MI), at a final concentration of $10 \mu \mathrm{l} /$ $\mathrm{ml}$, shown previously to elicit a maximal proliferative response by LPMC (20), was added such that the total volume per well was 200 $\mu$ l. Incubation was performed for $72 \mathrm{~h}$ at $37^{\circ} \mathrm{C}$ in a $5 \% \mathrm{CO}_{2}-95 \%$ air humidified atmosphere. Control cultures were prepared in an identical manner except that the mitogen was omitted. $6 \mathrm{~h}$ before harvest, 1 $\mu \mathrm{Ci}$ of $\left[{ }^{3} \mathrm{H}\right]$ thymidine (specific activity, $3 \mathrm{Ci} / \mathrm{mM}$; Becton-Dickinson \& Co., Schwartz/Mann Div., Orangeburg, NY) was added to each well. Cells were collected using a MASH II harvester (Microbiological Associates, Bethesda, MD) and $\left[{ }^{3} \mathrm{H}\right]$ thymidine uptake was measured as cpm in a Beckman LS7500 liquid scintillation system.

Target antigens. Gut-derived antigens were isolated from murine intestine, as previously described, through mechanical separation of gut epithelial cells, solubilization of epithelium-associated macromolecules in a PBS $/ 10^{-4} \mathrm{M}$ EDTA solution, and ethanol fractionation (12). Substances precipitating between 55 and $75 \%$ ethanol, upon resolubilization in PBS, dialysis against deionized water and lyophilization, were designated as epithelial cell-associated components (ECAC); materials derived from small bowel (ECAC-SB) and colon (ECAC-C) were separately processed and tested. Precipitation by ethanol as well as other biochemical separation procedures (below) were carried out at $4^{\circ} \mathrm{C}$, and all buffers contained $0.5 \mathrm{mM}$ phenylmethyl-sulfonyl fluoride, $8 \mathrm{mM}$ benzamidine (both from Sigma Chemical Co., St. Louis, MO), and $1 \mathrm{U} / \mathrm{ml}$ aprotinin (Mobay Chemical Corp., New York, NY) to inhibit proteases. Further, by both hemagglutination inhibition and double diffusion in agar, ECAC-SB and ECAC-C were shown not to contain components immunologically similar to the enterobacterial common antigen of Kunin; the latter, studied in a highly purified form as described (21), was a kind gift of Dr. Hubert Mayer, Max Planck Institute, Freiburg, Federal Republic of Germany.

In order to examine immune reactivity to individual components within ECAC, ECAC-SB was fractionated by preparative polyacrylamide gel electrophoresis. Eight- and three-tenths mg of ECAC-SB, dissolved in $3.0 \mathrm{ml}$ of saline $(5 \% \mathrm{vol} / \mathrm{vol}$ in glycerol), was electrophoresed on a nondenaturing $10 \%$ polyacrylamide slab gel, $\mathrm{pH} 8.8$, using a Bethesda Laboratory model V161 apparatus (Bethesda Research Laboratories, Gaithersburg, MD). Electrophoresis was carried out at $25^{\circ} \mathrm{C}$ at 25 $\mathrm{mA} / \mathrm{gel}$ until the bromphenol blue marker reached the end of the gel $(\sim 5 \mathrm{~h})$. Using a vertical $1.0-\mathrm{cm}$ gel strip developed with Coomassie Brilliant Blue G250 quick stain as a guide to band location (22), gel sections (designated P1 through P6, generally containing one component each) were sliced out, minced, and eluted into PBS by mechanical stirring overnight at $4^{\circ} \mathrm{C}$. Each supernatant was subsequently ultracentrifuged $(65,500 \mathrm{~g}, 45 \mathrm{~min})$, dialyzed against deionized water, and lyophilized. The purity of each gel-eluted component was assessed by re-electrophoresis on an analytical 10\% polyacrylamide gel (Fig. 2), and immune reactivity as well as antigenic specificity defined both by passive hemagglutination inhibition as well as by immunodiffusion (described in reference 12).

Elicitation of specific antisera. Antibody specific for ECAC-SB and ECAC-C was obtained through subcutaneous injection of $500 \mu \mathrm{g}$ of each antigen, dissolved in saline, and emulsified in an equal volume of Freund's complete adjuvant (250 $\mu \mathrm{g}$ Mycobacterium tuberculosis type $\mathrm{H} 37 \mathrm{Ra}$ ) (Difco Laboratories, Detroit, MI) into the hind footpads of New Zealand White rabbits. A panel of sera at peak titer was subsequently selected for study by a quantitative hemagglutination assay (9) and antigenic specificity was evaluated by radial immunodiffusion and hemagglutination inhibition (10).

Lymphocyte-mediated cytotoxicity. For this assay, LPMC and their subpopulations were isolated from inflammatory bowel disease and control patients as described above. Additional control mononuclear cells were isolated from the peripheral blood of a healthy individual by density gradient centrifugation over Ficoll-Hypaque. Viability of the latter cells was $>95 \%$ in all cases, and platelets were depleted by low-speed centrifugation in medium. For all cytotoxicity experiments, media was RPMI 1640 supplemented with 5\% heat-inactivated FCS, $100 \mathrm{U} / \mathrm{ml}$ penicillin, and $100 \mu \mathrm{g} / \mathrm{ml}$ streptomycin.

Study antigens were bound to 3-d-old chicken erythrocytes by a chromic chloride technique (23). Briefly, $40 \mu \mathrm{l}$ of antigen $(7 \mathrm{mg} / \mathrm{ml})$ was gently vortexed for 1 min with $25 \mu$ l of packed erythrocytes. After the addition of $175 \mu \mathrm{l}$ of saline, $250 \mu \mathrm{l}$ of a $0.075 \%$ chromic chloride solution $\left(\mathrm{A}_{590}=0.32\right)$ was added dropwise over $30 \mathrm{~s}$, the mixture gently vortexed for $4 \mathrm{~min}$, and the reaction stopped by dilution with $20 \mathrm{vol}$ of ice-cold saline. Adequacy of labeling was quantitated by a hemagglutination assay (9) utilizing hyperimmune sera with an established titer to each study antigen. As control antigen from a nonintestinal source, aqueous-soluble components of murine kidney, isolated by the PBS/EDTA extraction and ethanol fractionation procedure above, were utilized. Antigen-labeled erythrocytes were incubated with 100 $\mu \mathrm{Ci}$ of $\mathrm{Na}_{2}\left({ }^{51} \mathrm{Cr}\right) \mathrm{O}_{4}(295 \mathrm{mCi} / \mathrm{mg})$ (ICN Pharmaceutical Corp., Irvine, CA) for $\mathbf{4 0} \mathrm{min}$, washed three times, and dispensed in triplicate into wells of a 96-well microtiter plate to achieve a final density of $5 \times 10^{4}$ cells/well. LPMC, isolated as described above, were added just before target cells. The resultant final volume was $200 \mu \mathrm{l}$, and the effector to target $(\mathrm{E} / \mathrm{T})$ ratios were $10: 1-1: 10$. Cultures were incubated at $37^{\circ} \mathrm{C}$ for $4 \mathrm{~h}$ in $95 \%$ air-5\% $\mathrm{CO}_{2}$. The percentage of ${ }^{51} \mathrm{Cr}$ released into the medium was used as a measure of cell injury. For actual assays, background release from labeled erythrocytes averaged $6 \%$ or less over $4 \mathrm{~h}$. The mean $\mathrm{cpm}$ were determined for each triplicate set, and cytotoxicity (percent specific release) was calculated by the formula: $\left({ }^{\mathrm{cpm}}\right.$ experimental - ${ }^{\mathrm{cpm}}$ control)/( ${ }^{\mathrm{cpm}}$ total - ${ }^{\mathrm{cpm}}$ control) $\times 100$, where ${ }^{\mathrm{cpm}}$ experimental are supernatant counts in wells containing test LPMC and targets; and ${ }^{\mathrm{cpm}}$ control are the supernatant counts in wells containing control mononuclear cells and targets. ${ }^{\mathrm{cpm}}$ Total are the counts released when the standard number of target cells $\left(5 \times 10^{4}\right)$ were lysed in the presence of $5 \%$ Triton $\mathrm{X}-100 .{ }^{51} \mathrm{Cr}$ released by LPMC with the indicator cell alone (chicken erythrocytes without antigen labeling), monitored responses directed at red blood cell surface antigens in each case, and this, when detected, was subtracted in calculating the percent specific release. LPMC lysis of kidney (control) antigen-labeled indicator cells was monitored and separately tabulated. Targets exposed to medium alone were also routinely run as controls. However, since their ${ }^{51} \mathrm{Cr}$ release did not exceed background, these control data have not been included here.

Antibody-dependent cellular cytotoxicity. Recent reports from our laboratory, using peripheral blood mononuclear cells from Crohn's disease patients, suggest that anti-ECAC cytotoxicity can be antibody mediated (12). Therefore, we tested whether LPMC were also able to effect antibody-dependent cellular cytotoxicity against ECAC. We employed a procedure identical to that described above for lymphocytemediated cytotoxicity, except that heat-inactivated $\left(56^{\circ} \mathrm{C}, 30 \mathrm{~min}\right)$ patients' sera were diluted in medium and pipetted into wells to achieve a final concentration of 1:20,1:200, and 1:2,000. The formula for percentage specific release was the same as above, although ${ }^{\mathrm{cm}}$ experimental now designated supernatant counts in wells containing test serum in addition to LPMC and targets, and ${ }^{\mathrm{cpm}}$ control specified wells containing control serum, LPMC, and targets. Tested at the same time as controls were known ECAC-specific positive and negative rabbit sera, which had an established titer by hemagglutination to each study antigen of $1: 4,000$ and $<4$, respectively.

Inhibition of the cytotoxic response by antigen. Two million LPMC in $500 \mu \mathrm{l}$ of medium were preincubated with 2 or $8 \mu$ l of inhibitor (ECAC or kidney antigen at $7 \mathrm{mg} / \mathrm{ml}$, or saline) for $1 \mathrm{~h}$ at $37^{\circ} \mathrm{C}$. After three washings $(2,000, \mathrm{~g}, 4 \mathrm{~min}$ each), LPMC were added to antigenlabeled target cells in the standard cytotoxicity assay. Percent inhibition was calculated by the formula $1-$ ( $^{\mathrm{cpm}}$ experimental/ ${ }^{\mathrm{cpm}}$ control) $\times 100$, as described previously (24). That these inhibition experiments were carried out in antigen excess was verified through measurement of the capacity of the culture supernate to inhibit a standard anti-ECAC serum, utilizing a technique (passive hemagglutination) developed for glycoproteins and having a sensitivity approaching that of radioimmunoassay in our hands (10). In control experiments assessing nonspecific effects of ECAC upon antigen-receptor binding, addition of 2 or $8 \mu \mathrm{l}$ of ECAC to dilutions $(1: 16,1: 128,1: 512)$ of anti-BSA serum did not reduce that serum's titer against BSA-labeled erythrocyte indicator cells, compared with 2 or $8 \mu \mathrm{l}$ of saline. Hence, inhibition 
detected with addition of ECAC was regarded as antigen-specific and not due to a nonspecific interference with antigen-antibody binding.

Statistical methods. The data were analyzed using statistical techniques which accounted for multiple measurements per individual and were specific to the individual study designs of the various experiments. For Tables I, IV, and V, the kidney antigen response was first subtracted from the two other measurements on each subject and then the Hotelling-Lawley Trace (25), a multivariate test statistic, was used to test for the effect of case-control status on the resultant bivariate response. Table VI also employed the Hotelling-Lawley Trace, after adjusting for individual subjects, to test for effects on the bivariate ECAC-C, ECAC-SB response. Adjustment for the multiple testing situation in making comparisons between various subgroups within one experiment was accomplished through the use of the Student Newman-Keul procedure (26) with a significance level of 0.05 . Only in the case of count data (Results, first section) were data log transformed in order to stabilize variances before using an analysis of variance.

\section{Results}

LPMC: recovery, composition, and response to mitogen. By the techniques described above and summarized in Fig. 1, histologically normal intestinal mucosa yielded $10^{7} \mathrm{LPMC} /$ gram of wet tissue, while mucosa involved by chronic inflammatory bowel disease had a 2-4-fold higher yield, in agreement with earlier observations (8). Viability was $\geq 85 \%$ for cells from both diseased and normal intestinal segments. Cell composition of purified, unfractionated LPMC was $57 \pm 12 \%$ T lymphocytes, by OKT 3-positive staining; $18 \pm 9 \%$ B lymphocytes, by cell surface immunoglobulin fluorescence; and $10 \pm 6 \%$ macrophages, by the presence of diffuse, coarse, nonspecific esterasepositive granules. As previously reported, no significant differences were observed in the relative proportions of $T$ and non$T$ cells isolated from diseased and control tissues $(27,28)$.

Control LPMC from all but one patient demonstrated a $\geq 26,000 \mathrm{cpm}$ incorporation of $\left[{ }^{3} \mathrm{H}\right]$ thymidine (mean $\pm \mathrm{SD}$, $59,200 \pm 34,000$ ) in the presence of $10 \mu \mathrm{g} / \mathrm{ml}$ of PHA for $72 \mathrm{~h}$, the mean uptake being comparable between tumor-bearing patients and those with benign disease. Similarly, LPMC from all but one patient with chronic inflammatory bowel disease incorporated $\geq 24,000 \mathrm{cpm}$ of $\left[{ }^{3} \mathrm{H}\right]$ thymidine (mean $\pm \mathrm{SD}$, $86,933 \pm 63,400)(P>0.07$, comparing control and disease LPMC).

Cell-mediated cytotoxicity of LPMC for epithelial antigens. ECAC-specific cytotoxicity of LPMC isolated from seven patients with chronic inflammatory bowel disease was significantly greater $(P<0.02)$ than that found with control LPMC (Table I). Test antigen derived from small intestine (ECAC-SB) and colon (ECAC-C) epithelium were both reactive, compared with control kidney antigen prepared by techniques (solubilization in EDTA/PBS, ethanol fractionation) identical to those used for isolation of ECAC. LPMC from five of seven patients responded to both ECAC preparations. The mean $\pm S D{ }^{51} \mathrm{Cr}$ release for the seven control subjects was $0.03 \pm 0.08 \%$ (ECACC) and $0.40 \pm 1.05 \%$ (ECAC-SB) (Table I).

The influence of the effector to target $(E / T)$ ratio was examined in preliminary experiments with LPMC from four patients (Fig. 3). ECAC-specific cytotoxicity was detectable and near-maximal at an E/T of one whether unfractionated or T lymphocyte-enriched LPMC were used. Reactivity to colon and small bowel-derived ECAC behaved similarly in this regard. Subsequently, unless otherwise stated, cytotoxicity assays were carried out at an E/T of one. The studies which follow were generally performed with inflammatory bowel disease (and not control) LPMC, with the goal of characterizing the ECAC-reactive cell.

Antibody independence of the LPMC cytotoxic response. To determine the importance of serum-derived antibody, the LPMC anti-ECAC cytotoxic response was evaluated in the presence of varying dilutions of autologous (inflammatory bowel disease) sera. LPMC, isolated from three inflammatory bowel disease patients known reactive with ECAC and incubated with a high dilution of serum $(1: 2,000)$, demonstrated ECAC-specific cytotoxicity comparable with that of LPMC alone (13.3 \pm 5.8 vs. $16.5 \pm 9.8$ for ECAC-C; $5.5 \pm 2.6$ vs. $6.2 \pm 6.5$ for ECAC-SB). Similarly, at higher serum concentrations (1:200, 1:20), LPMC cytotoxicity did not increase (mean lysis $<16.5 \%$ for ECAC-C, $<6.2 \%$ for ECAC-SB).

Because patient sera may have lacked relevant antigen-
I

CELL ISOLATION

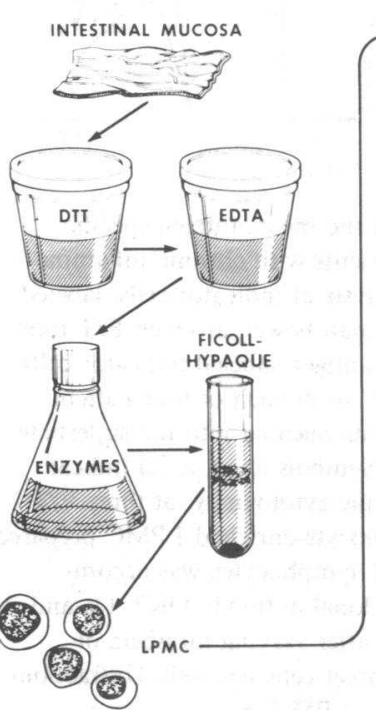

II

CELL FRACTIONATION
III

CYTOTOXICITY ASSAY

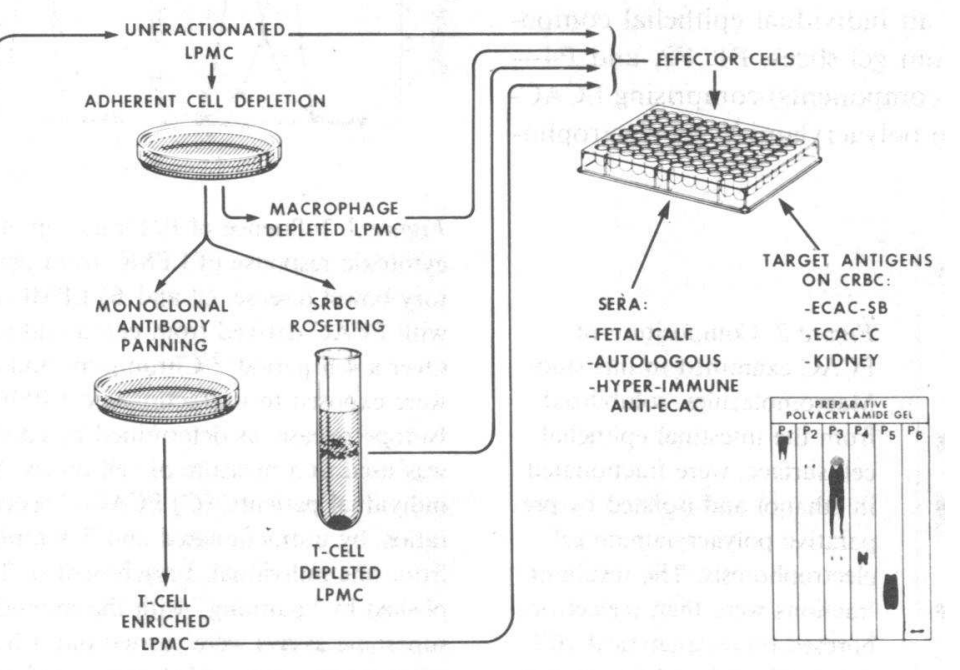

IPMC
Figure 1. Scheme for the isolation, fractionation, and testing of LPMC for their cytotoxic potential against intestinal ECAC. LPMC were released from surgically resected intestine by enzymatic means followed by density gradient centrifugation and enrichment/depletion techniques for subset isolation. After the addition of varying numbers of effector cells to medium with sera, a standard number $\left(5 \times 10^{4} /\right.$ well) of labeled target cells were added to measure antigenspecific reactivity. DTT, dithiothreitol. 
Table I. Lytic Effect of Unfractionated LPMC Upon Indicator Cells Labeled with ECAC Derived from Colon and Small Bowel As Well As with Control Kidney Antigen

\begin{tabular}{llll}
\hline & \multicolumn{2}{l}{ Percent specific lysis* } & \\
\cline { 2 - 4 } Source of effectors & ECAC-C & ECAC-SB & $\begin{array}{c}\text { Kidney } \\
\text { antigen }\end{array}$ \\
\hline $\begin{array}{l}\text { Inflammatory } \\
\quad \text { bowel disease }\end{array}$ & & & \\
$\quad \begin{array}{l}\text { All } \\
\text { Responders only" }\end{array}$ & $12.5 \pm 8.9$ & $7.1 \pm 6.5$ & $0.8 \pm 1.1$ \\
$\begin{array}{l}\text { Controls } \\
\text { All }\end{array}$ & $0.03 \pm 0.08$ & $0.40 \pm 1.11$ & $0.29 \pm 0.76$ \\
& & & \\
\end{tabular}

* Shown are the mean \pm SD specific cytotoxic responses of LPMC from seven disease and seven control subjects. All assays were performed at an $\mathrm{E} / \mathrm{T}$ cell ratio of one.

$\ddagger P<0.02$ by multivariate analysis (Hotelling-Lawley Trace), comparing ECAC-specific lysis of inflammatory bowel disease and control LPMC.

$\S$ Inflammatory bowel disease LPMC reactivity against ECAC was significantly different from zero (ECAC-C, $P=0.04$; ECAC-SB, $P=0.03$ ).

"LPMC from five of seven patients responded to both ECAC-C and ECAC-SB.

specific immunoglobulin, the cytotoxic potential of patient LPMC was further assessed in the presence of heterologous hyperimmune ECAC-specific sera (titer by passive hemagglutination $>1: 4,000$ ). At a time when the positive control (normal peripheral mononuclear cells) demonstrated an antibody-dependent cytotoxic response to ECAC-C (13.9 $44.3 \%)$ and to ECAC-SB $(25.7 \pm 10.4)$ in the presence of a 1:200 dilution of hyperimmune-specific sera, eight preparations of LPMC, whether from histologically normal (four) or diseaseinvolved intestine, were not augmented by serum (mean increase in cytotoxicity $<0.9 \pm 0.6$ ). These data would indicate that antibody-dependent ECAC-specific cytotoxicity is not an important mechanism at the gut level under these conditions.

Cytotoxicity with components of ECAC. To assess whether additional antigen purification would reveal the LPMC reactivity to be highly-specific for an individual epithelial component, five macromolecules (from gel slices, P1, P2, and P4one component each; $\mathrm{P} 3$, two components) comprising ECACSB and isolated by preparative polyacrylamide gel electropho-

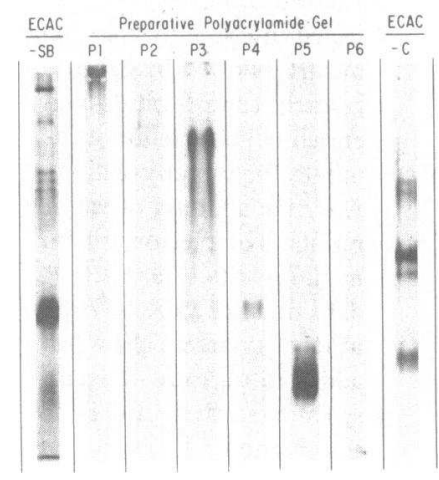

Figure 2. Composition of ECAC examined in this study. Macromolecules, solubilized from the intestinal epithelial cell surface, were fractionated in ethanol and isolated by preparative polyacrylamide gel electrophoresis. The resultant fractions were then reelectrophoresed on an analytical $10 \%$ polyacrylamide gel under nondenaturing conditions. resis (Fig. 2), were each evaluated as targets for cytotoxicity with LPMC from three additional patients with active chronic inflammatory bowel disease (Table II). Adequate and consistent component-target cell binding was verified in passive hemagglutination by taking each labeled target cell type against a panel of standard rabbit antisera developed to each component. LPMC cytotoxicity against the ECAC-SB component isolated from gel slice P1 was, in general, 2-3-fold higher compared with other purified macromolecules derived from small intestinal epithelium, although all reactive LPMC demonstrated a significant level of cytotoxicity to each component above control (mean $+2 \mathrm{SD}=2.6 \%$ ).

Effector cell responsible for anti-ECAC reactivity. In initial experiments to characterize the nature of the lamina propria cell causing ECAC-specific cytotoxicity, adherent cells were markedly depleted from LPMC ( $10 \pm 6 \%$ reduced to $2 \pm 1.5 \%)$ by the plastic adherence technique. Compared with unfractionated LPMC, the ECAC-specific cytotoxicity of adherent cell-depleted preparations was retained or enhanced (Table III), suggesting that the cytotoxic cell was nonadherent.

Therefore, T lymphocyte-enriched LPMC were prepared from six additional patients, using a panning technique with an established $T$ lymphocyte-specific monoclonal antibody (OKT 11), and assayed for ECAC-specific reactivity. Markedly enhanced cytotoxicity to both ECAC-C and ECAC-SB was demonstrated by the cell preparations enriched in $\mathrm{T}$ lymphocytes $(96 \pm 1.7 \% \mathrm{~T}$ lymphocytes) compared with unfractionated LPMC $(P<0.05)$ (Table IV). T lymphocyte-depleted LPMC $(23 \pm 8 \% \mathrm{~T}$ lymphocytes) retained cytotoxic potential but were not significantly different from unfractionated LPMC ( $P$ $>0.30$ ). Use of an alternative $T$ lymphocyte-specific monoclonal antibody (OKT 3) for cell fractionation of LPMC from two additional patients gave similar findings: a fourfold rise in ECAC-C specific cytotoxicity, comparing unfractionated LPMC

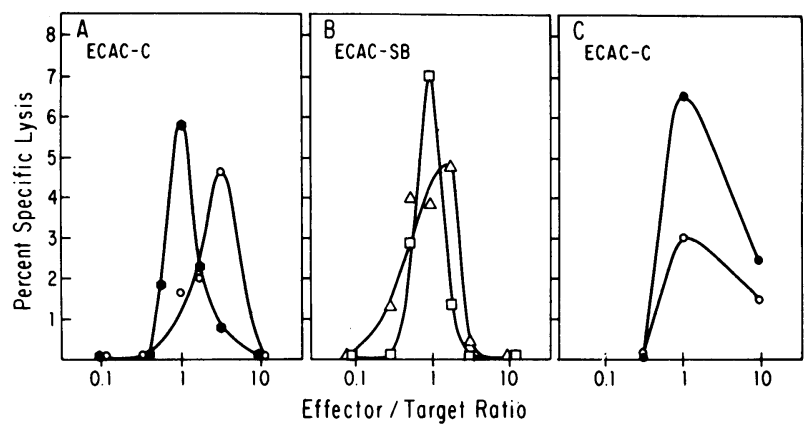

Figure 3. Influence of $\mathrm{E} / \mathrm{T}$ ratio upon the mean antigen-specific cytotoxic response of LPMC from patients with chronic inflammatory bowel disease. ( $A$ and $B$ ) LPMC lysis of indicator cells, labeled with ECAC derived from colon and small bowel, at seven E/T ratios. Over a 4-h period, ${ }^{51} \mathrm{Chromium}$ - and antigen-labeled indicator cells were exposed to unfractionated LPMC from each of four patients. Isotope release, as determined by counts released into the supernate, was used as a measure of cell injury. Symbols $(0, \bullet, \Delta, \square)$ indicate individual patients. $(C)$ ECAC-C specific cytotoxicity, at three $\mathrm{E} / \mathrm{T}$ ratios, by unfractionated and T lymphocyte-enriched LPMC prepared from one individual. Enrichment in T lymphocytes was accomplished by "panning" with the monoclonal antibody OKT 11 , and supernate assays were carried out $4 \mathrm{~h}$ after varying numbers of effector cells were added to $5 \times 10^{4}$ target cells per well. Unfractionated LPMC, o; T lymphocyte-enriched LPMC, ๑. 
Table II. Level of Reactivity to ECAC by LPMC from Chronic Inflammatory Bowel Disease Patients*

\begin{tabular}{lll}
\hline & \multicolumn{2}{l}{ Percent specific lysisł } \\
\cline { 2 - 3 } Type of antigen & All patients & Responders only§ \\
\hline $\begin{array}{l}\text { ECAC-SB gel-purified } \\
\text { subfraction }\end{array}$ & & \\
P1 & & \\
P2 & $6.0 \pm 6.9$ & $9.1 \pm 6.4$ \\
P3 & $2.4 \pm 2.1$ & $3.6 \pm 0.2$ \\
P4 & $3.0 \pm 2.7$ & $4.5 \pm 1.2$ \\
Kidney control antigen & $3.3 \pm 4.2$ & $5.0 \pm 4.2$ \\
& $0.7 \pm 1.1$ & $1.1 \pm 1.4$ \\
\end{tabular}

* Utilizes ECAC-SB subfractions isolated by preparative gel electrophoresis.

$\ddagger$ Shown is the mean \pm SD for the specific cytotoxic response. Assays were carried out at an E/T ratio of 10 . In addition to controls, kidney antigen and unlabeled chicken erythrocytes, a limit of four test antigens could be studied per assay for comparison.

$\S$ Number of patients studied was three, of whom two responded to a specific ECAC-SB derived component at the level of $\geq 1 \%$ specific lysis. Polyacrylamide gel fractions P5 and P6 (dye front) were not evaluated.

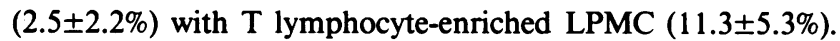
Reactivity to kidney control antigen remained low with all cell preparations (Table IV). This same analysis, when carried out at an E/T ratio of 10 , showed similar results (data not shown). These experiments suggested that a $T$ lymphocyte population was responsible for cytotoxicity to ECAC.

$\mathrm{T}$ lymphocytes remaining in the $\mathrm{T}$ lymphocyte-depleted fractions $(23 \pm 8 \%)$ may have accounted for residual reactivity to ECAC in the experiments above. Therefore, a SRBC rosetting technique was used to further reduce the percentage of $T$ lymphocytes in LPMC from four additional patients known to be reactive with ECAC. Compared with unfractionated LPMC, T lymphocyte-depleted preparations $(11.6 \pm 5.6 \% \mathrm{~T}$ lymphocytes) showed a significant reduction in ECAC-specific cytotoxicity $(P<0.05)$ (Table V). This effect was evident at an $\mathrm{E} / \mathrm{T}$ ratio of 10 (shown in the table) and one (data not shown).

Table III. Characteristics of the ECAC-specific Cytotoxic Cell: Nonadherence to Plastic Surfaces*

\begin{tabular}{lll}
\hline & \multicolumn{2}{l}{ Percent specific lysis $¥$} \\
\cline { 2 - 3 } Source of effector cells§ & ECAC-C & ECAC-SB \\
\hline Unfractionated LPMC & $6.5 \pm 9.2$ & $3.8 \pm 3.3$ \\
Adherent cell-depleted LPMC & $6.3 \pm 4.9$ & $8.5 \pm 4.4$
\end{tabular}

* LPMC, purified by density gradient centrifugation of intestinal mucosal cells released after collagenase treatment, were tested for ECAC-specific cytotoxicity before and after a 120-min incubation at $37^{\circ} \mathrm{C}$ on plastic Petri dishes. Adherence reduced the percentage of macrophages from a mean of $10 \%$ (unfractionated LPMC) to $2 \%$ (macrophage-depleted LPMC), as assessed by nonspecific esterase staining.

$\ddagger$ Data are shown as the mean \pm SD specific cytotoxic response of LPMC for the indicated antigens at an E/T ratio of 1.

$\S \mathrm{LPMC}$ were from four patients with chronic inflammatory bowel disease.
Table IV. LPMC Reactivity with ECAC* $\ddagger$

\begin{tabular}{lccc}
\hline & \multicolumn{2}{l}{ Percent specific lysis } & \\
\cline { 2 - 4 } Effector cells & ECAC-C & ECAC-SB & $\begin{array}{c}\text { Kidney } \\
\text { antigen }\end{array}$ \\
\hline Unfractionated & $5.0 \pm 1.9$ & $2.2 \pm 2.2$ & $0.8 \pm 1.6$ \\
T lymphocyte enriched & $19.0 \pm 9.9^{\| \prime}$ & $20.9 \pm 11.4^{\| \prime}$ & $1.9 \pm 1.4$ \\
T lymphocyte depleted & $7.0 \pm 7.1$ & $4.6 \pm 4.8$ & $1.3 \pm 2.8$
\end{tabular}

* Using T lymphocyte-enriched and depleted effector cells prepared by monoclonal antibody panning.

$\ddagger 20 \times 10^{6}$ adherent cell-depleted LPMC were incubated for $30 \mathrm{~min}$ at $4^{\circ} \mathrm{C}$ with a 1:100 dilution of OKT 11 monoclonal antibody. After thorough washing, the cells were dispersed slowly onto a goat antimouse Ig-coated plate. After $1 \mathrm{~h}$ at $4^{\circ} \mathrm{C}$, adherent and nonadherent cells were separately removed and assessed for purity by immunofluorescence.

$\S$ Data from six patients are expressed as mean \pm SD of a 4-h assay carried out in triplicate at an $\mathrm{E} / \mathrm{T}$ ratio of one.

"At the 0.05 level, reactivity of T lymphocyte-enriched LPMC toward ECAC-C and ECAC-SB was significantly different from that of unfractionated and T lymphocyte-depleted LPMC.

Because NK cells may copurify with $T$ lymphocytes on the basis of similar cell surface markers (receptor for sheep erythrocytes, and glycoprotein detected by OKT 3), we depleted NK cells from LPMC of four additional ECAC-reactive patients in order to assess the contribution of NK to ECAC-specific cytotoxicity. Compared with unfractionated LPMC (4.5 $\pm 0.4 \%)$, nonadherent (NK-depleted) cells retained cytotoxicity toward ECAC-SB (3.9 \pm 1.3$)$. Similar results were found with ECAC-C $(6.6 \pm 2.5 \%$ and $4.7 \pm 4.1 \%$ for unfractionated and NK-depleted LPMC). Immunofluorescence with an NK-specific monoclonal antibody (Leu 11b) showed that NK cells constituted a small percentage $(1.3 \pm 1.1 \%)$ of mononuclear cells among LPMC that was further reduced in the depleted (nonadherent) fraction $(0.5 \pm 0.7 \%)$. The number of cells recovered in the adherent fraction (generally $<10^{6}$ ) was too small to allow an adequate evaluation of their cytotoxic potential.

Effect of preincubation with ECAC. To further assess the antigenic specificity of the cytotoxic response of both unfrac-

Table V. Anti-ECAC LPMC Reactivity Before and After T Lymphocyte Depletion by the SRBC-Rosetting Technique*

\begin{tabular}{|c|c|c|c|}
\hline \multirow[b]{2}{*}{ LPMC } & \multicolumn{3}{|c|}{ Percent specific lysisł } \\
\hline & ECAC-C $\S$ & ECAC-SB & $\begin{array}{l}\text { Kidney } \\
\text { antigen }\end{array}$ \\
\hline Unfractionated & $11.8 \pm 5.5$ & $2.5 \pm 3.7$ & $0.4 \pm 0.5$ \\
\hline T lymphocyte depleted & $0.6 \pm 0.8$ & $<0.2$ & $<0.2$ \\
\hline
\end{tabular}

* $5 \times 10^{6}$ adherent cell-depleted LPMC in $1 \mathrm{ml}$ RPMI 1640 were mixed with an equal volume of $1 \%$ sheep erythrocytes, spun at $200 \mathrm{~g}$ for $5 \mathrm{~min}$, and incubated for $1 \mathrm{~h}$ at $4^{\circ} \mathrm{C}$. After gentle resuspension and density gradient centrifugation, cells at the interface were washed and assessed for cell type by immunofluorescence.

‡ Mean specific lysis \pm SD was for triplicate cultures of LPMC exposed to antigen-labeled indicator cells for a period of $4 \mathrm{~h}$ at an E/T ratio of 10 .

$\S$ At the 0.05 level, reactivity of unfractionated LPMC toward ECAC$\mathrm{C}$ was significantly different from that of $\mathrm{T}$ lymphocyte-depleted LPMC. Statistical significance was not reached with respect to ECAC-SB. 
tionated and T lymphocyte-enriched preparations, LPMC from four patients were preincubated with study antigens as inhibitors (ECAC-C, ECAC-SB, and kidney antigen) or saline for $1 \mathrm{~h}$, extensively washed, then assayed for residual reactivity to ECAC-labeled target cells by the standard cytotoxicity assay (Table VI). Both LPMC preparations (unfractionated and T lymphocyte-enriched), upon exposure to soluble ECAC-C or ECAC-SB, demonstrated a marked reduction in ECAC-specific reactivity compared to those incubated with saline. Incubation with control kidney antigen did not, in general, reduce LPMC cytotoxicity. Percent inhibition after exposure to ECAC, calculated as described earlier (24), was $>82 \%$ in each instance, and exceeded $94 \%$ in six of the eight conditions tested. ECAC alone had no nonspecific inhibitory effect upon binding of antibody to antigen-labeled target cells when evaluated in other antigen-antibody systems (see Methods).

Correlation of cytotoxicity with clinical status. By clinical diagnosis, $72 \%$ of ulcerative colitis patients and $68 \%$ of Crohn's disease patients possessed ECAC-reactive LPMC; in $>80 \%$ of the cases, reactivity was found to ECAC-C and ECAC-SB simultaneously. The magnitude of the ECAC-specific response in six ulcerative colitis patients with severe disease was 7.2 \pm 5.4 and $5.3 \pm 4.3$ (ECAC-SB and ECAC-C, respectively), compared with $1.9 \pm 2.3$ and $4.3 \pm 5.2$ in the four patients with moderate disease. Activity of disease was moderate in all but one Crohn's disease patient. Parenteral steroids did not abrogate cytotoxicity (24/27 positive) in patients receiving them (27/31). Because the origin of LPMC in this study was consistently the large bowel in ulcerative colitis (12/12) and small bowel in Crohn's disease (17/19), an analysis of the effect of LPMC location upon cytotoxicity within each disease category (ulcerative colitis, Crohn's disease) could not be undertaken.

\section{Discussion}

Previous studies have demonstrated that functional LPMC may be prepared from the intestinal mucosa (6-8). In extending these studies, we sought to develop a method by which antigen-

Table VI. Effect of Preincubation of LPMC with Study Antigens Upon the Antigen-specific Cytotoxic Response*

\begin{tabular}{llcc}
\hline & & \multicolumn{2}{c}{ Percent specific lysisł } \\
\cline { 3 - 4 } Source of effectors & Inhibitors & ECAC-C & ECAC-SB \\
\hline Unfractionated & Saline & $5.2 \pm 1.2$ & $3.0 \pm 2.0$ \\
& ECAC-C & $0.2 \pm 0.4 \S$ & $<0.2 \S$ \\
& ECAC-SB & $<0.2 \S$ & $0.5 \pm 0.6 \S$ \\
& Kidney antigen & $3.4 \pm 2.4$ & $4.5 \pm 3.0$ \\
T lymphocyte enriched & Saline & $17.3 \pm 9.3$ & $19.5 \pm 15.5$ \\
& ECAC-C & $0.8 \pm 1.1 \S$ & $0.2 \pm 0.4 \S$ \\
& ECAC-SB & $3.7 \pm 3.3 \S$ & $0.2 \pm 0.2 \S$ \\
& Kidney antigen & -11 & $19.0 \pm 19.2$
\end{tabular}

* $2 \times 10^{6}$ LPMC were incubated for $1 \mathrm{~h}$ in $0.5 \mathrm{ml}$ of RPMI 1640 containing $30 \mu \mathrm{g}$ of antigen $(0.015 \mathrm{mg} / \mathrm{ml})$ or saline, washed, and assayed for antigen-specific cytotoxicity in the standard manner $(E / T$ ratio equal to one).

¥ Data are given as mean \pm SD of LPMC from four patients individually analyzed in triplicate. Cell suspensions prepared by monoclonal antibody panning contained $96 \% \mathrm{~T}$ lymphocytes.

§ At the 0.05 level, cytotoxicity of ECAC-C and ECAC-SB pretreated LPMC was significantly different from that of saline-treated LPMC.

"Not done. specific responses of LPMC isolated from chronically inflamed mucosa might be characterized, particularly in regard to their reactivity with the recently described soluble epithelial cellassociated components (12). Our ultimate goal was to test the hypothesis that patients with chronic idiopathic inflammatory disease of mucosal epithelium have undergone auto-sensitization.

Circulating and mucosa-derived antibodies and mononuclear cells with specificity for intestinal epithelium have been observed by workers in several gastrointestinal diseases over the last $20 \mathrm{yr}(3,29-32)$, but little attempt has been made to isolate and characterize the relevant epithelium-associated antigens. While the presence within the intestinal mucosa of organ- and region-specific antigens has been inferred indirectly from immunofluorescence studies utilizing heterologous absorbed sera (33), crude cell homogenates have been the principal substrate for examining the issue of autoimmunogenicity (34, 35). Recent work demonstrating that ECAC themselves, free of other portions of the intestinal cell wall (lamina propria, serosa, and muscle), could elicit organ-specific inflammation upon injection with adjuvant (36), encouraged us to focus more specifically upon these cell-associated macromolecules.

Evidence that we found by microcytotoxicity assay an antigen-specific response unique to patients with chronic inflammatory mucosal disease (Crohn's disease and ulcerative colitis) is as follows. Reactivity of patient LPMC could be blocked in the same assay system by preincubation with soluble antigen. Second, responses directed against only the indicator cell (chicken erythrocyte) in the absence of test antigen, when monitored simultaneously in each assay, were insignificant. Third, patients with other intestinal diseases, including malignant and nonmalignant conditions, did not demonstrate an immune response to epithelial cell-associated antigens when studied concomitantly as disease-controls. Comparable degrees of PHA-driven $\left[{ }^{3} \mathrm{H}\right]$ thymidine uptake indicated that the lack of antigen-specific reactivity by control LPMC was not due to an unusual degree of functional cell suppression. Fourthly, reactivity to ECAC was evident with highly purified macromolecules, particularly component $\mathrm{P} 1$, which were shown to be homogeneous under nondenaturing conditions by analytical polyacrylamide gel electrophoresis. Interestingly, immune responsiveness appeared to be directed to more than one component (Table II). From their differing $\boldsymbol{R}_{\mathrm{f}}$ values (Fig. 2 ), and their individual reactivity with determinant-specific sera (12), we assume that each " $P$ " component is a different macromolecule. One could speculate that a common sensitizing determinant is shared among several epithelial cell-associated components, or that an autoimmune process at the mucosal surface is directed, for reasons not yet understood, to several distinct antigens, a concept more broad than that which we generally envision in experimental models of autoimmune disease (experimental allergic encephalomyelitis, for instance) $(37,38)$. Examples of this broad reactivity to self include the autoimmune hemolytic anemias (I, i, P, and Rh-related antigens) and systemic lupus erythematosis (native double-stranded DNA and the phospholipid cardiolipin) in man, as well as experimental autoimmune orchitis (aspermatogenic proteins AP1, AP2, GP1, and GP4) in the guinea pig (39-42).

The cytotoxic response that we found should be considered in the context of the murine origin of the study antigens. The presence of species-specific major histocompatibility loci antigens in our preparation cannot be ruled out, but seems unlikely 
in view of: $(a)$ the tight association of these antigens with cell membranes (43); cells and membranes were pelleted out of the starting material in the current study; $(b)$ the poor aqueoussolubility of histocompatibility antigens when they have been mobilized from cell membranes (44); $(c)$ the absence of patient reactivity to murine kidney antigens similarly prepared by dissociation and ethanol fractionation; and $(d)$ the absence of reactivity by LPMC from control patients to these same murine-derived fractions. No immunological cross-reactivity was found between ECAC and the enterobacterial common antigen of Kunin (see Methods), making it less likely that LPMC cytotoxicity is directed toward bacterial antigens. However, Kotz and Roche (45) have recently reported the isolation from human colon of components quite similar to murinederived ECAC; immunological identity was shown by a passive hemagglutination inhibition technique, and individual macromolecules demonstrated similar migratory rates $\left(R_{f}\right)$ by polyacrylamide gel electrophoresis.

Cytotoxic capabilities of LPMC explored in previous work all evaluated nonspecific killing. In general, no differences have been found between LPMC derived from chronic inflammatory bowel disease and control tissue $(7,46)$, although Shorter et al. (47) recently reported antibody-dependent cellular cytotoxicity and spontaneous cell-mediated cytotoxicity against autologous epithelial cells using, respectively, LPMC from patients with inflammatory bowel disease and other disorders. In contrast, our data suggest, for the first time, that LPMC are capable of antigen-specific cytotoxicity and that this phenomenon is confined to mononuclear cells derived from inflammatory bowel disease-involved intestine, as opposed to cells isolated from histologically normal mucosa. Furthermore, our study shows that cytotoxicity apparently requires specific antigen recognition as a necessary step before destruction of ECACcoated targets. The lack of effect of adherent cell removal from the effector population, the inability of patients and hyperimmune sera to enhance the degree of killing, the increase in cytotoxicity by $T$ cell enrichment and its loss by $T$ cell depletion, all indicate that $\mathrm{T}$ lymphocytes are the responsible cells. The possibility that residual NK cells are causative of a portion of the cell lysis is highly unlikely, considering that depletion of this cell subset did not significantly alter LPMC cytotoxicity against ECAC-coated targets. In addition, spontaneous cell-mediated cytotoxicity has not been demonstrated for LPMC $(7,46,48)$, and treatment of LPMC with interferon does not induce cytotoxicity against K562 target cells (Fiocchi, C., R. R. Tubbs, and K. R. Youngman, submitted for publication). We are uncertain why an $\mathrm{E} / \mathrm{T}$ ratio of one was nearoptimal in our system, except that the further addition of a particular $T$ cell subset may result in overall suppression of antigen-specific responses. This is partly supported by our recent observation that the degree of mitogen-induced proliferation of a mixture of peripheral blood mononuclear cells and LPMC is progressively suppressed as the proportion of LPMC is increased (49). It should be noted that, since we were unable to obtain LPMC from inflamed mucosa of disease other than chronic inflammatory bowel disease, we cannot rule out the possibility that LPMC reactivity to ECAC may be related to disruption of the intestinal epithelial cell barrier. Evidence that a local immunological autosensitization is the primary process will likely depend upon studies of ECAC reactivity among affected and nonaffected members of families with a genetic predisposition to chronic inflammatory bowel disease, and upon investigation in animals intentionally sensitized to intestinal epithelial antigens.

Differences in immune mechanisms for the anti-ECAC response in the intestinal mucosa (reported here as $T$ lymphocyte mediated) compared with that in the peripheral circulation (reported in reference 12 as antibody mediated) deserves comment. In one scheme, B and T lymphocytes, sensitized to antigen within Peyers' patches, could each achieve a different distribution in lymphoid tissue upon their obligatory systemic migration before homing to receptors on high endothelial gut venules (50). Antigen-specific $\mathrm{T}$ lymphocytes, in contrast to other immune cells, may be rather completely recruited back to inflamed mucosa, perhaps because of their involvement in the tissue-destructive response, accounting for their ready detection there and not in the peripheral blood (12). Alternatively, T lymphocytes specific for ECAC may become activated and numerically expand primarily in mucosa, in response to continued exposure there to the sensitizing antigen.

Detection of LPMC reactivity to epithelial cell-associated components, isolated under conditions not requiring cell homogenization with release of intracellular contents, encourages use of these readily obtainable, presumably native macromolecules to explore further the immunochemistry and the immunoregulation of antigen-specific autoimmune phenomena directed toward mucosal epithelium in both man and in the experimental animal.

\section{Acknowledgments}

We wish to gratefully acknowledge the aid of Dr. Elizabeth Delong (Cancer Center, Duke University Medical Center) in the statistical analysis of our data.

This investigation was supported by U. S. Public Health Service grants AM 27260 and AM 30399, awarded by the National Institute of Arthritis, Metabolism, Digestive and Kidney Diseases, Department of Health and Human Services, and by grants from the National Foundation for Ileitis and Colitis, Inc.

\section{References}

1. Bookman, D. E., and M. D. Cooper. 1973. Pinocytosis by epithelium associated with lymphoid follicles in the bursa of Fabriscius, appendix, and Peyer's patches. An electron microscopic study. Am. J. Anat. 136:455-477.

2. Owen, R. L. 1977. Sequential uptake of horseradish peroxidase by lymphoid follicle epithelium of Peyer's patches in the normal unobstructed mouse intestine: an ultrastructural study. Gastroenterology. 72:440-451.

3. Perlmann, P., and O. Broberger. 1963. In vitro studies of ulcerative colitis. II. Cytotoxic action of white blood cells from patients on human fetal colonic cells. J. Exp. Med. 117:707-715.

4. Watson, D. W., A. Quigley, and R. J. Bolt. 1966. Effects of lymphocytes from patients with ulcerative colitis on human adult colon epithelial cells. Gastroenterology. 51:985-993.

5. Stobo, J. D., T. B. Tomasi, K. A. Huizenga, R. J. Spencer, and R. G. Shorter. 1976. In vitro studies of inflammatory bowel disease: surface receptors of the mononuclear cell required to lyse allogeneic colonic epithelial cells. Gastroenterology. 70:171-176.

6. Bull, D. M., and M. A. Bookman. 1977. Isolation and functional characterization of human intestinal mucosal lymphoid cells. J. Clin. Invest. 59:966-974.

7. MacDermott, R. P., G. O. Franklin, R. M. Jenkins, I. J. Kodner, G. S. Nash, and I. J. Weinrieb. 1980. Human intestinal mononuclear cells. I. Investigation of antibody-dependent, lectin-induced and spontaneous cell-mediated cytotoxic capabilities. Gastroenterology. 78:4756.

8. Fiocchi, C., J. R. Battisto, and R. G. Farmer. 1979. Gut mucosal 
lymphocytes in inflammatory bowel disease. Isolation and preliminary functional characterization. Dig. Dis. Sci. 24:705-714.

9. Roche, J. K., E. D. Day, and H. D. Hill. 1978. Rabbit antibodies to ovine submaxillary mucin: detection, specificity and cross-reactivity. Immunochemistry. 15:339-343.

10. Roche, J. K., V. A. Varitek, H. D. Hill, and E. D. Day. 1979. Specificity and $\mathrm{T}$ lymphocyte dependence of the humoral immune response in the rat to purified ovine and porcine mucins. Mol. Immunol. 16:609-619.

11. Roche, J. K., S. L. Cook, and E. D. Day. 1981. Goblet cell glycoprotein: an organ-specific antigen for gut. Isolation, tissue localization and immune response. Immunology. 44:799-810.

12. Aronson, R. A., S. L. Cook, and J. K. Roche. 1983. Sensitization to epithelial antigens in chronic mucosal inflammatory disease. I. Purification, characterization, and immune reactivity of murine epithelial cell-associated components (ECAC). J. Immunol. 131:2796-2804.

13. Comer, G. M., W. G. Romey, D. P. Kotler, and P. R. Holt. 1983. Isolation and preliminary characterization of colonic immunocompetent cells in the acquired immunodeficiency syndrome. Gastroenterology. 84:1129a. (Abstr.)

14. Truelove, S. C., and L. J. Witts. 1955. Cortisone in ulcerative colitis: final report of a therapeutic trial. Br. Med. J. 2:1041-1048.

15. Schachter, H., and J. B. Kirsner. 1975. Definitions of inflammatory bowel disease of unknown etiology. Gastroenterology. 68:591600.

16. deDombal, F. T., I. C. Burton, and S. E. Clamp. 1974. Shortterm course and prognosis of Crohn's disease. Gut. 15:435-443.

17. Li, C. Y., L. T. Yam, and K. W. Lam. 1970. Acid phosphatase iso-enzyme in human leukocytes in normal and pathological conditions. J. Histochem. Cytochem. 18:473-481.

18. Wysocki, L. J., and V. L. Sato. 1978. "Panning" for lymphocytes: a method for cell selection. Proc. Nat'l. Acad. Sci. USA. 75:28442848.

19. Kaplan, M. E., and C. Clark. 1974. An improved rosetting assay for detection of human $\mathrm{T}$ lymphocytes. J. Immunol. Methods. 5:131-135.

20. Fiocchi, C., J. R. Battisto, and R. G. Farmer. 1981. Studies on isolated gut mucosal lymphocytes in inflammatory bowel disease. Detection of activated $\mathrm{T}$ cells and enhanced proliferation to Staphylococcus aureus and lipopolysaccharides. Dig. Dis. Sci. 26:728-737.

21. Mannel, D., and Mayer, H. 1978. Isolation and chemical characterization of the Enterobacterial common antigen. Eur. J. Biochem. 86:361-370.

22. Reisner, A. T. P., P. Wemes, and C. Bucholtz. 1975. The use of Coomassie Brilliant Blue G250 perchloric acid solution for staining in electrophoresis and isoelectric focusing on polyacrylamide gels. Anal. Biochem. 64:509-516.

23. Gold, E. R., and H. H. Fudenberg. 1967. Chromic chloride: a coupling reagent for passive hemagglutination reaction. J. Immunol. 99:859-866.

24. Grol, M., and K. Schumacher. 1983. Purification and biochemical characterization of human liver-derived inhibitory protein (LIP). J. Immunol. 130:323-326.

25. Morrison, D. F. 1976. Multivariate Statistical Methods. MoGrawHill Book Co., Inc., New York. Second ed. 5:223-224.

26. Miller, R. G. 1981. Simultaneous Statistical Methods. SpringerVerlag Publishing Co., New York. Second ed. 2:81-90.

27. Selby, W. S., G. Janossy, M. Bofill, and D. P. Jewell. 1984. Intestinal lymphocyte subpopulations in inflammatory bowel disease: an analysis by immunohistochemical and cell isolation techniques. Gut. 25:32-40.

28. Bartnik, W., S. G. ReMine, M. Chiba, W. R. Thayer, and R. G. Shorter. 1980. Isolation and characterization of colonic intraepithelial and lamina proprial lymphocytes. Gastroenterology. 78:976985.

29. Langercrantz, R., S. Hammerstrom, P. Perlmann, and B. E. Gustafsson. 1968. Immunological studies in ulcerative colitis. IV. Origin of auto-antibodies. J. Exp. Med. 128:1339-1352.
30. Das, K. M., R. Dubin, and T. Nagai. 1978. Isolation and characterization of colonic tissue-bound antibodies from patients with idiopathic ulcerative colitis. Proc. Nat'l. Acad. Sci. USA. 75:45284532.

31. Nagai, T., and K. M. Das. 1981. Detection of colon antigen(s) in tissues from ulcerative colitis using purified colitis colon tissuebound IgG (CCA-IgG). Gastroenterology. 81:463-470.

32. Shorter, R. G., D. B. MoGill, and R. C. Bahn. 1984. Cytotoxicity of mononuclear cells (MC) for autologous colonic epithelial cells (ACEC) in colonic diseases. Gastroenterology. 86:13-22.

33. Rabin, R. S. 1976. Duodenum-, ileum- and colon-specific antigens. Int. Arch. Allergy Appl. Immunol. 50:133-141.

34. Richardson, E. S., and S. Leskowitz. 1961. An attempt at production of autoimmunity to tissue of the gastrointestinal tract. Proc. Soc. Exp. Biol. Med. 106:357.

35. Kirsner, J. B., J. G. Elchlepp, M. B. Goldgraber, J. Abaze, and H. Ford. 1959. Production of an experimental ulcerative colitis in rabbits. Arch. Pathol. 68:392-408.

36. Roche, J. K., S. R. Cook, and E. D. Day. 1981. Cellular cytotoxicity and gastrointestinal inflammation in inbred rats: induction with gut organ-specific antigens. Immunology. 44:489-497.

37. Kies, M. W. 1977. Experimental allergic encephalomyelitis. 1973. In Biology of Brain Dysfunction. G. E. Gaull, editor. Plenum Press, New York. 1:185.

38. Braum, P. E., S. W. Brostaff. 1977. Proteins of myelin. In Myelin. P. Morcel, editor. Plenum Press, New York. 1:201.

39. Leddy, J. P., and S. N. Swisher. 1978. Acquired immune hemolytic disorders. In Immunological Diseases. M. Samter, editor. Little, Brown and Co., Boston. 1:1187-1189.

40. Shoenfeld, Y., J. Rauch, H. Massicotte, S. K. Datta, J. AndreSchwartz, B. D. Strollar, and R. Schwartz. 1983. Polyspecificity of monoclonal lupus autoantibodies produced by human-human hybridomas. N. Engl. J. Med. 308:414-420.

41. Hagopian, A., J. J. Jackson, D. J. Carlo, G. A. Limjuco, and E. H. Eylar. 1975. Experimental allergic aspermatogenic orchitis. III. Isolation of spermatozoal glycoproteins and their role in allergic aspermatogenic orchitis. J. Immunol. 115:1731-1743.

42. Teuscher, C., G. C. Wild, and K. S. K. Tung. 1983. Acrosomal autoantigens of guinea pig sperm. I. The purification of an aspermatogenic protein, AP2. J. Immunol. 130:317-322.

43. Walsh, F. S., and M. J. Crumpton. 1977. Orientation of cellsurface antigens with lipid bilayer of lymphocyte plasma membrane. Nature (Lond.). 269:307-311.

44. Cresswell, P., and J. R. Dawson. 1975. Dimeric and monomeric forms of HLA antigens solubilized by detergent. J. Immunol. 114:523525.

45. Kotz, K., and J. K. Roche. 1984. Human colonic glycoprotein: isolation and partial characterization of epithelial cell-associated components. Gastroenterology. 86:1352.

46. Chiba, M., W. Bartnik, G. G. ReMine, W. R. Thayer, and R. G. Shorter. 1981. Human colonic intra-epithelial and lamina proprial lymphocytes. Cytotoxicity in vitro and the potential effects of the isolation methods on their functional properties. Gut. 22:177-186.

47. Shorter, R. G., D. B. McGill, and R. C. Bahan. 1984 Cytotoxicity of mononuclear cells for autologous epithelial cells in colonic diseases. Gastroenterology. 86:13-22.

48. Falchuk, Z. M., E. Barnhard, and I. Machado. 1981. Human colonic mononuclear cells: studies of cytotoxic function. Gut. 22:290294.

49. Fiocchi, C., K. R. Youngman, and R. G. Farmer. 1983. Immunoregulatory function of human intestinal mucosa lymphoid cells: evidence for enhanced suppressor cell activity in inflammatory bowel disease. Gut. 24:692-701.

50. Butcher, E. C., R. G. Scollay, and I. L. Weismann. 1980. Organ specificity of lymphoid migration: mediation by highly selective lymphocyte interaction with organ-specific determinants on high endothelial venules. Eur. J. Immunol. 10:556-561. 